\title{
Le drame de l'autisme: entre amour et désir
}

\section{The drama of autism: between love and desire}

Jacques Cabassut*1

Céline Barriol*2

A partir de leur praxis, les auteurs proposent de questionner l'impensable de la problématique autistique avec pour point de départ l'amour et le désir, qui en est son grand véhicule. A cet effet, ils proposent de mettre en rapport sujet de l'individuel et sujet du collectif, formation de la foule et passion mélancolique et ce, afin d'en saisir les enseignements sur les effets de structuration du sujet dit autiste dans ses rapports avec l'Autre, et réciproquement, sur la qualité du lien social contemporain. Ils argumentent que la conjoncture entre la problématique autistique et le discours capitaliste potentialise la mélancolie du soignant [en miroir à la mélancolisation de l'Autre maternel]. La conception actuelle du soin, qui évince la spécificité de l'amour de transfert spécifique à la souffrance autistique, détruit toute idée d'implication du professionnel dans la rencontre. Dès lors, seule la restauration des dimensions de l'amour de transfert et du désir, comme le traitement de leur incurable, permettent de penser et/ou de repenser les pratiques actuelles, cette fois à partir de l'éthique propre à la clinique psychanalytique.

Mots clés: Amour, autisme, désir, lien social

${ }^{* 1,2}$ Université Nice Sophia Antipolis (Nice, France). 
Les histoires d'amour finissent mal en général ! ...

Les Rita Mitsouko

Seul l'amour permet à la jouissance de condescendre au désir. Jacques Lacan, Séminaire Livre X, l'Angoisse, leçon du 13 mars 63.

\section{Dramaturgie clinico-sociale}

L'amour, et le désir qui en est son grand véhicule, sont convoqués ici comme point d'appui à la clinique analytique face à l'impensable de la souffrance autistique et aux conceptions neurocognitives ${ }^{1}$ qui alimentent sa molle désignation actuelle de "trouble du spectre autistique" (TSA). C'est peu dire en effet, que le modèle scientiste du "pathos" à l'oeuvre, dit modèle "bio-psycho-social", n'est aucunement une réactualisation de la cause des névroses freudiennes même si facteur biologique, facteur phylogénétique et facteur psychologique sont évoqués (Sauret, 2008, p. 52). ${ }^{2}$

${ }^{1}$ Le débat actuel sur l'utilisation précoce de la tablette numérique auprès des enfants en bas âge en est la parfaite illustration; celle-ci, qui s'entend essentiellement comme stimulation cognitive dans le rapport à l'objet, prive le sujet d'un contact à l'altérité humaine nécessaire à sa construction subjective dans la "parolisation" du réel et de son nécessaire support (empathie, affects, réponses non attendues d'autrui au lieu de la répétition du même de la machine etc.), renforçant par là-même — c'est un comble! — l'activité auto-érotique.

2 "Le facteur psychologique réside dans le conflit qui surgit entre le moi et le ça (et non dans les capacités cognitives): impossible en effet d'échapper radicalement aux pulsions internes. Le facteur phylogénétique désigne le caractère biphasé de l'activité sexuelle humaine, inexistant dans les espèces animales (et non les déterminations 


\section{ARTIGOS}

L'amour en effet, à le nouer de surcroît à la problématique autistique, pose à l'homme une énigme incommensurable, si archaïque qu'elle s'affiche comme une évidence, un besoin "primaire", auquel chacun voue un véritable culte, prêt à sacrifier à sa croyance, comme à se mettre en quête incessante et passionnelle de sa présence ou de son moindre signe. Ainsi, la néoténie du petit homme implique-t-elle que la valeur de l'objet (d'amour) qui seule est à même de le protéger contre les dangers et de remplacer la vie intra-utérine perdue, en est fortement augmentée. "Le facteur biologique, conclut Freud, à la fois, est à l'origine des premières situations de danger, et crée le besoin d'être aimé qui n'abandonnera plus l'être humain". Lacan, précisera quant à lui les conditions de la seconde naissance, celle symbolique au langage, à travers ce nouage du stade du miroir à la symbolisation primordiale (Sauret, 2008, p. 52). Bref, c'est dire si la réintroduction de l'amour dans le champ de l'autisme - comme inversement! — est au carrefour des problématiques des constructions subjectives, des pratiques cliniques qui empruntent ou pas à l'amour de transfert, comme de la qualité du lien social actuel annexés aux discours dominants qui la déterminent: Lacan dans sa leçon du 6 janvier 1972, ne confrontait-il pas le discours capitaliste à ses effets de "forclusion de la castration, de rejet des affaires du sexe et des choses de l'amour"?

Ainsi en va-t-il du traitement de l'Hilflosigkeit, de la détresse et de l'angoisse sociale dans la construction subjective. ${ }^{3}$ Freud dans son "Malaise",

sociales et culturelles): les revendications pulsionnelles de la sexualité infantile menacent d'entraîner dans le refoulement les motions sexuelles ultérieures de la puberté. Le facteur biologique désigne non pas un élément organique mais une des conditions majeures de la vie du nouveau-né: précisément l'état de détresse (Hilflosigkeit) et de dépendance très prolongée du petit de l'homme" (Sauret, 2008).

${ }^{3} \mathrm{Tel}$ que bon nombre d'auteurs l'ont remarquablement mis en évidence, la valeur de notre existence - tel Winnicott via le sentiment d'existence, la transitionnalité ou le "good enough mother", ou Spitz dans le "syndrome d'hospitalisme" - , ne nous est pas donnée naturellement, "instinctuellement" à la différence de l'animal. Nous ne sommes ni sûrs de notre valeur, ni de notre existence "réelle". Si nous avons besoin d'être aimé, c'est bien parce que nous ne sommes pas des monades autosuffisantes (Pépin, 2018, p. 116), mais que nous naissons à notre vérité dans la néoténie, ce lien de dépendance à l'Autre et aux autres. Bref, "nous avons besoin d'être aimés pour compenser cette détresse infantile, dont nous réussirons peut-être à sortir, mais en en payant le prix. 'Nous serons les victimes de cette victoire' écrit Louis Althusser dans l'avenir dure longtemps" (Pépin, 2018, p. 16). 
parle du surgissement d'angoisse chez le petit enfant, confronté au risque de la perte d'amour de l'autre dont il est dépendant; celle-ci ne peut pas être autre chose qu'une angoisse sociale (Freud, 1939/2001, p. 67). Ce qui corrobore donc, que la psychanalyse n'est pas seulement une méthode dédiée à la souffrance personnelle ou à la psychologie individuelle, mais qu'elle traite également du lien social et de ses maux, le sujet de l'individuel comme celui du collectif se confondant dans le traitement de la pulsion (Cabassut, 2017). ${ }^{4}$ Mieux ou pire, pour Freud, la foule représente le premier état de 1 'humain. Dès lors, contrairement à l'idée reçue que la psychologie des foules est une généralisation de la psychologie individuelle, c'est bien le contraire qui se produit (André, 2015, pp. 64 et 66). ${ }^{5}$ Que peut bien dès lors nous enseigner la formation de la foule sur la structuration autistique, et réciproquement?

Justement: la question de l'amour, dans ses dimensions relationnelles comme via la notion "d'état amoureux", est au fondement de la formation de la foule comme de celle du sujet, dans ses effets de puissance de suggestion hypnotique de massification, d'unification ${ }^{6}$ et d'idéalisation de l'être et/ou de l'être-ensemble [pour emprunter à la terminologie d'A. Arendt]. Ainsi donc, les identités ${ }^{7}$ [massificatrices et fascisantes] de "Foule, Patrie, 700 Peuple" (André, 2015, p. 59) de "race", de communautés, comme les identités

${ }^{4}$ Freud (1939/2001, p. 85) nous l'enseigne en soulignant que "le développement de la culture ressemble tant à celui de l'individu et travaille avec les mêmes moyens".

${ }^{5}$ L'unique différence entre le petit enfant et les adultes, “(...) se limite à ceci que la communauté plus vaste des hommes vient en lieu et place du père ou des deux parents" (Freud, Ibid.).

${ }^{6}$ D'où leurs effets de "fascisation": Littéralement le fascisme, consiste à réunir dans un seul faisceau, à atteindre la force dans l'unité compacte. Le fascio (ou faisceau) n'est autre que le symbole de l'autorité des licteurs dans la Rome antique (source: Aux origines du fascisme, Le club de Mediapart).

${ }^{7}$ Le rapport au Miroir constitue partout l'un des paradigmes fondamentaux de l'identité, soit du rapport narcissique à son image dans le mouvement simultané de reconnaissance et de séparation propre à la formule même du principe d'identité: "A est A", "où le verbe être constitue une médiation entre deux termes identiques" (Benslama, 2014, p. 144). Une image qu'il aimera d'amour, l'enfant se prenant pour elle, en faisant le noyau de son moi (Ricoeur, 2007, p. 9) alors qu'elle n'est que le support de son être. L'échec du rapport au Miroir, à l'aliénation à l'image spéculaire, comme à la virtualité du signifiant du fait des problématiques autistiques dans le rapport à l'existence et au langage, composera la pathologie autistique et un enseignement du champ des identités. 


\section{ARTIGOS}

individuelles et subjectives qui les composent, trouvent leur fondement premier dans le lien d'amour à l'Altérité première, ${ }^{8}$ déterminante dans le rapport à soi, au monde et aux autres. A ce titre, Anzieu (1981) évoque que la formation $\mathrm{du}$ groupe peut constituer une enveloppe psychique; Klein (1955/1968) la rapporte quant à elle à la notion de noyau autistique propre à chacun d'entre nous, laquelle devient identité adhésive dans sa forme paroxystique selon Haag (1995).

Pourtant, si la souffrance autistique a maintes fois fait l'objet de remarquables travaux autour du pivot de la jouissance, du rapport à l'objet [objet voix ou constitution d'objet complexe], du rapport à l'Autre premier (soit la Chose), peu à notre connaissance, portent sur l'amour comme point d'entrée, hormis évidemment, ceux désignant la "fiction d'un narcissisme primaire" (Maleval, 2009, p. 39). Et pour cause: "nous sommes enseignés par ceux - les autistes - qui témoignent de notre être dans son rapport originel à la langue, inaccessible et pourtant infiniment présent, actif pour chacun de nous, ce c'ur d'être sans Autre encore, sans représentation, identification de quoi et de qui que ce soit". L'Autre de l'amour ne "consiste" pas, $\mathrm{du}$ fait que "l'Autre en tant que matière discursive n'existe pas (...). D'être sans Autre n'est pas être hors langage (...)" pour autant (Chaumon, 2009, pp. 109 et 110). Ainsi, à suivre Frances Tustin, l'angoisse archaïque corrélée à un sentiment impensable de séparation propre à la dépression primitive autistique, s'avère dans sa forme ultime de néantisation. Ces registres antérieurs au registre de l'inconscient, ${ }^{9}$ nous conduisent donc en ces temps de nécessités logiques de construction subjective d'un Autre pacificateur de jouissance. Ladite consistance peut se réaliser à la façon du psychosé [par introjection (Mélancolie) ou par projection (Paranoïa) d'un Autre malveillant; par "jointure" et "colmatage" langagier à 1'Autre (Schizophrénie - Rouzel, 2013, p. 30)] ou dans le style autistique, via cette tentative qui est la sienne de faire consister un "Autre non persécuteur" comme forme élémentaire de la

${ }^{8}$ Lacan distingue l'Autre [lieu de ses déterminations langagières, du "trésor de signifiants" antérieur à lui et lieu de la Loi, à laquelle il fait recours pour mettre de l'ordre dans le monde humain / Lapeyre \& Sauret, 2000, p. 60] de l'autre [comme alter ego et semblable].

9 'Un premier registre 'sensitif' serait celui de l'autisme précoce, inscrivant du matériel sensoriel chaotique. Un deuxième registre scriptural inscrirait des 'images' aux capacités langagières parfois prodigieuses antérieurs au registre de l'inconscient auquel l'enfant autiste n'accède pas" (Chaumon, 2009, p. 110). 
subjectivité $^{10}$ (Maleval, 2009, pp. 102-103). Dans tous les cas, il n'y a pas de savoir supposé à l'Autre [à l'instar de la névrose], mais constitution d'un lieu d'adresse possible et potentiel du transfert dans la rencontre, à condition que le semblable qui occupe ce lieu d'incarnation Autre, accuse réception de ce "savoir y faire avec la jouissance": "tout discours a une adresse et il faut que quelqu'un vienne incarner ce point d'amarrage" (Menard, 2008, pp. 17, 24 et 30$)$.

\section{Sujet de l’inconscient / Sujet du social politique}

En définitive, la souffrance autistique pose et repose l'insondable question de l'amour, du lien qui l'établit, et donc du (dé)nouage RSI [RéelSymbolique-Imaginaire] qui le sous-tend, jusqu'à en former dans notre emprunt, le plagiat du néologisme lacanien: "il n'y a pas de rapport amoureux" tout comme il n'y a pas de rapport sexuel. ${ }^{11}$ Lacan, en effet, face au ratage de structure du sexuel, qu'il lit dans l'impossible rapport entre les sexes, va convoquer l'amour comme suppléance au dit ratage, une suppléance à ce que le rapport sexuel échoue à faire: qu'à deux, ça fasse Un (Ricoeur, 2007, p. 6). L'on pourrait alors avancer que l'autiste est celui qui a raté le ratage de la division subjective: ni un, ni deux, ni avec, ni sans l'Autre. Pourrait-on pour autant avancer que l'autiste est celui qui n'a pas été suffisamment illusionné par l'amour de l'Autre? La réalisation d'un Autre de

10 “'A l'encontre de ce que l'on observe dans les psychoses, le double autistique n'est pas fondamentalement persécuteur, bien au contraire: le sujet trouve souvent en lui un élément propre à apaiser ses troubles. Dans l'autisme, le double est dans le réel, constataient les Lefort, 'mais peut faire séparation d'avec l'Aut'. Ce n'est pas un objet étrange et maléfique qui témoigne d'une délocalisation de la jouissance; c'est un objet familier, toujours maitrisé, ou considéré comme un 'ami' inhérent au monde sécurisé, et dont le sujet se sert volontiers pour traiter la jouissance pulsionnelle" (Maleval, 2009, p. 110).

${ }^{11}$ L'amour supplée au non rapport sexuel: aucun A(a)utre, aucun homme, aucun objet, aucune chose, ne pourra venir compenser le manque à être structurel et structurant du sujet, qui, du fait de sa prématurité néonatale, devra s'en remettre à l'Autre dans la régulation de sa détresse (Hilflosigkeit) structurale, comme dans le manque dans l'Autre du signifiant qui totaliserait son être. L'on saisit mieux alors l'apport humain de l'amour parallèlement à la notion animalière d'attachement. 


\section{ARTIGOS}

synthèse, caractéristique de l'amour de transfert de type autistique (Maleval, 2009) tendrait à le prouver. Du coup, à suivre cette piste, i.e que la souffrance autistique est prototypique de l'impuissance de l'amour à suppléer au réel, peut-être pouvons-nous le reporter comme caractéristique de la qualité contemporaine du lien social, laquelle se détermine de plus en plus sur un mode ségrégatif, plutôt que de l'amour porté [à l'Autre, à soi, et aux autres], dans son expression langagière. L'effondrement de l'illusion fondamentale de l'amour et du désir, tant du côté du sujet que de l'Autre, ne produit-il pas une mélancolisation du lien (social), déshumanisante pour tout un chacun, ["autiste", parent, éducateur, soignant] dans le rapport à un autre, alors désigné comme pur objet de soin [à éduquer, à réeduquer, à contrôler]? A ce titre, l'on pourrait se demander si ce ratage caractéristique, n'est pas paradigmatique d'une mélancolisation du lien social post-moderne, tel que le discours capitaliste $^{12} l^{\prime}$ 'illustre?

\section{Désamour et/ou non amour?}

L'originalité d'une relation [d'amour] de transfert de type "autistique", "passe par le double (et) s'avère possible à certaines conditions, tandis qu'un mode d'interprétation orienté par le traitement de l'Autre (...)" est caractéristique de la non moins originalité du "fonctionnement subjectif des autistes" (Maleval, 2009, p. 14). C'est bien à travers l'objet, voire donc l'objectivation de l'Autre, que l'amour de transfert pourra se réaliser. L'objet-voix et l'objet - regard y tiennent à ce titre, une place centrale: "la voix est surtout ce qui dans la parole, fait autorité indépendante de la signification de ce qui est énoncé - , ce qui commande une exaltation particulière ou un ébranlement intime de l'auditoire. La parole convainc, en faisant tomber les résistances, les inhibitions et les censures, mais la voix, elle, commande au-delà de la conviction qui a pu entraîner la parole. Car ce que recherche Hitler ce n'est pas simplement l'adhésion d'un grand nombre d'individus à ses propres idées ou à son propre fantasme: c'est la foule, qui, réunit et soudée en un véritable corps, ${ }^{13}$ doit donner les manifestations du symptôme qui découle de son propre fantasme". Ce en quoi, comme l'affirme André (2015, pp. 61-62),

${ }^{12}$ Se reporter à Lacan (1969-1970) et Sauret (2009).

${ }^{13}$ Souligné par nous. 
le nazisme est le triomphe de la psychologie de la foule sur la psychologie du sujet [triomphe donc de l'identité adhésive dans ses effets de massification].

Ainsi donc, l'objet-voix est au centre, mieux au croisement du fonctionnement des foules et de la souffrance autistique. L'objet-voix, objet pulsionnel freudien, se décline sous la forme de l'objet a dans sa conceptualisation lacanienne. ${ }^{14}$ Elle est ce qui porte, supporte et "trans-porte" l'amour au sein des relations précoces mère-enfant. C'est elle qui "trans-faire" et métaphorise donc la rencontre amoureuse, qui s'incarne dans le babil ou lalangue lacanienne. La parole, supportée par cet objet pulsionnel qu'est la voix, spécifie la pathologie dans cette carence énonciative, cette singulière difficulté à prendre la position d'énonciateur: "Quand le sujet autiste cherche à communiquer, il le fait autant que possible d'une manière qui ne met en jeu ni sa jouissance vocale, ni sa présence, ni ses affects" (Maleval, 2009, p. 77). Il faut dire que la voix, en tant qu'objet de jouissance, par rapport aux autres objets pulsionnels oral, anal et scopique, possède le privilège de commander à "l'investissement du langage", lequel permet de structurer le monde des images et des sensations de l'infans; en tant que tel, la voix n'est aucunement sonorité de la parole mais "ce qui porte la présence du sujet dans son dire". En rejetant toute dépendance à l'égard de l'Autre, le sujet autiste ne s'aliène pas aux lois langagières, pour conserver la terrible liberté de ne pas aliéner son être dans le langage (Maleval, 2009, pp. 78-81).

\section{Synthèse et rebond}

L'intrication entre la pulsion et l'amour est problématiquement à l'épicentre de notre questionnement, comme à celui de Freud qui, au sein de sa "Psychologie des foules...", via l'ambiguiité de la notion d'objet, ${ }^{15}$ en vient à

${ }^{14}$ L'autiste ne place pas sa voix dans le vide de l'Autre ce qui lui permettrait de s'inscrire sous le signifiant unaire de l'identification primordiale. Il faut dire que la voix comme objet pulsionnel, n'est pas du registre du sonore mais s'avère hors sens (Maleval, 2009 , p. 81), tout comme le regard est hors-cadre du champ de vision.

${ }^{15}$ Le mécanisme qui lie la foule au meneur présente un schéma qui vaut aussi bien pour l'état amoureux que pour la formation de la foule, un objet venant se mettre à la place de l'idéal du moi. Cependant, contrairement aux thèses de Gustave Lebon sur lesquelles il s'appuie, Freud amalgame dans un premier temps deux tendances via le 


\section{ARTIGOS}

fusionner objet pulsionnel et personne aimée. ${ }^{16}$ Freud en effet, dans le texte, les amalgame et les confond pour mieux les distinguer ensuite, marquant la nécessité de les concevoir dans une forme de dialectique permanente et inextricable: objet pulsionnel et objet d'amour entremêlent jouissance, amour et désir.

Il résoudra la difficulté du nouage en affirmant que le phénomène de foule s'apparente plus à l'hypnose qu'à la relation amoureuse: ${ }^{17}$ la relation hypnotique est en fait un comble de la relation amoureuse, comportant à la fois un abandon amoureux illimité et une exclusion complète de la satisfaction sexuelle; surtout, il ne s'agit pas du même objet dans l'hypnose [l'hypnotisé n'est pas captivé par l'image de l'hypnotiseur mais le pouvoir de l'objet voix et/ou regard], ou dans la relation amoureuse [l'amoureux est fasciné par l'image de l'objet aimé] (André, 2015, pp. 70-71). Bref, c'est donc bien l'objet pulsionnel [objet a] ${ }^{18}$ et non l'objet narcissique de l'amour [i(a)] qui caractérise le phénomène de foule, dans la provocation de la confusion entre cet objet réel et le signifiant idéal [soit $\mathrm{S} 1$, où se repère le sujet, lequel forme également le secret du meneur de foules précise Serge André). C'est bien cette même confusion de l'idéal et de l'objet qui explique, alors que le meneur se présente via l'objet a comme le regard et la voix, que ce soit bien une relation d'amour qui s'établit entre lui et la foule, plutôt qu'une relation pulsionnelle

concept de libido [les pulsions et l'amour], qu'il avait précédemment différenciées. Qu'il s'agisse d'amour strictement sexuel, de celui porté aux idées abstraites, comme de l'amour de soi ou bien parental, Freud opère là une synthèse pulsionnelle d'un "pousse à l'union sexuelle". Pourtant, Freud introduit dans le texte deux objections, l'état amoureux (Verliebtheit) [qui désigne des pulsions d'amour dans la foule en tant qu'elles sont détournées de leur but sexuel d'origine], l'identification [présentant un autre type de lien sentimental également détaché du but sexuel], distinguant à nouveau le champ de la pulsion et de l'amour. Bref, Freud au terme de son raisonnement, doit scinder le concept de libido : la formation de la foule joue littéralement l'amour contre les pulsions (André, 2015, pp. 67-70)

${ }^{16}$ A propos de la notion freudienne d'objet, s'agit-il de l'objet de la pulsion, soit un objet réel que Lacan nomme objet a ou bien de l'objet d'amour, donc objet imaginaire, narcissique que Lacan écrit i(a)? (André, 2015, pp. 69-70).

17 "Alors que dans la relation amoureuse, l'objet est mis à la place du moi (ce que note exactement le mathème lacanien $\mathrm{i}(\mathrm{a})$, dans l'hypnose il vient à la place de l'idéal du moi" (André, 2015, p. 70).

${ }^{18}$ Dans le totalitarisme, en particulier dans l'hitlérisme, le führer occupe la place de l'objet a (André, Ibid). 
proprement dite, laquelle n'apparait qu'à certains moments sous forme d'angoisse (André, 2015, p. 71).

\section{Retour vers la Clinique de l'amour et du désir}

Comment saisir la combustion du pulsionnel et de l'amour, l'assemblage de "a" et i(a), la confusion de l'idéal et de l'objet? Sinon à travers la scène mythique du récit clinique de Lucien, qui est également la scène narrative d'une séance regroupant équipe d'IME et analyste des pratiques. Le savoir du mythos, propre à la clinique psychanalytique, tentera de rendre compte de la vérité de la souffrance autistique, autrement qu'au travers des prétentions objectivantes et statistiques de l'administration de la preuve du TSA. ${ }^{19}$

Lucien, 13 ans, a accompli des progrès considérables selon le dire de l'équipe, depuis son arrivée il y a trois ans à l'IME: il s'est progressivement mis à parler, tout en lâchant bon nombre de ses comportements archaïques [tel que celui de badigeonner quotidiennement les murs de sa chambre avec ses excréments, l'automutilation, la fréquence des passages à l'acte hétéro-agressifs durant lesquels "il cassait tout" (sic)]. Cet enfant continue néanmoins de questionner l'équipe dont il "casse" les membres, pour mieux les réparer: après les coups, les insultes et les crachats, viennent de façon compulsivement réparatrice, les locutions "aimer!", "Lucien aimer!" ou "Arrête Lucien!". Il les touche également autrement, cet enfant donnant l'impression de "souffrir tout le temps" (sic) pour l'équipe. Celle-ci, à l'instar de Lucien, est partagée dans une forme d'ambivalence "hainamorative". ${ }^{20}$ L'équipe hérite donc, par voie de transfert, ${ }^{21}$ de la problématique de Lucien. Laquelle, à vrai dire? Celle qui condense l'amour dans le désir parental.

19 “Les cas en psychanalyse sont des exemples de concept. Or, chacun sait qu'il n'y a pas d'exemple de concept. C'est pourquoi la démonstration ne démontre rien: c'est un acte narratif. C'est un acte car elle fait évènement dans le dénouement du transfert". (Khon, 1999, p. 52).

${ }^{20} \mathrm{~L}$ "'hainamoration" est un néologisme lacanien condensant la haine et l'amour quant à l'être "énamouré".

${ }^{21} \mathrm{Le}$ (mouvement de) transfert renvoie certes à la motion d'amour (le transfert étant de l'amour qui s'adresse à du savoir selon Lacan), donc à la dimension haineuse qui en est son envers. Il renvoie surtout, dans son partage du "trans", à ce transport entre deux êtres qui modifie les deux êtres en présence. 


\section{ARTIGOS}

Reprenons: ce dont l'équipe atteste, c'est bien d'une (re)naissance à l'humanité de Lucien de par la qualité de la clinique socio-éducative et thérapeutique réalisée. Il faut entendre là, le fait que le petit homme procède toujours d'une double naissance, biologique mais aussi symbolique, dans le désir de l'Autre [parental]. Tout enfant nécessite une adoption d'amour au sein du désir parental. Ce que le syndrome d'hospitalisme, dans son envers catastrophique, nous révèle.

Ce moment mythique de nouage de l'amour et du désir s'inscrit sur une scène traumatique du roman familial de Lucien. Adopté dans un orphelinat d'un pays de l'Est, ses parents rêvent d'une fille, blonde comme la femme qui deviendra sa mère. L'instant du regard porté sur Lucien, ne précipite pas le désir du temps pour comprendre. C'est monsieur qui réalisera l'effectuation de la hâte, celle du moment de conclure dans un "véritable coup de foudre" (sic) de cet enfant handicapé: " $j$ 'ai décidé d'adopter cet enfant" dira-t-il. La fixation traumatique qui en résulte ne cesse pas de se répéter sur les uns et les autres: sa mère fera une sévère dépression, les parents se déchireront sur la responsabilité de la souffrance autistique de Lucien, se séparant finalement. Quant à lui, il ne cesse pas de ne pas écrire l'impossible "hainamoration" [Se faire aimer / Se faire rejeter] dont il est l'objet, reposant sans cesse la question de son adoption dans le désir de l'Autre, autrement dit comme tout un chacun, l'énigme de son origine. L'équipe en reparle car depuis son dernier séjour en famille, les comportements violents se sont accentués, ainsi que ceux issus de l'élan hainamoratif ["quand il fait des câlins, il nous pince en même temps" (sic)], ce qui a pour effet de cliver l'équipe entre les tenants du travail éducatif et ceux portant sur le soin.

\section{Conclusion de transfert}

Quitte à parler d'amour, parlons de transfert, qui est véritable amour, lequel permet le miracle thérapeutique de Lucien et autorise la renaissance au Désir de l'Autre. Tel est le fondement du transfert selon Freud (1914/1953 et 1939/2001) lequel est à la fois moteur et résistance dans la cure comme dans la relation clinique et ce, pour la même raison :

$1^{\circ}$ L'agir de transfert [agieren] prend le relais de l'indicible comme de l'ineffable, ce en quoi il permet d'atteindre au souvenir intraduisible car trop chargé d'affects, immémorable car tombant sous le coup de l'amnésie infantile. Il est alors le point d'appui, particulièrement comme nous l'avons vu en ce qui concerne la souffrance autistique, d'autant plus dans ce qui permet 
la réactualisation des "impressions fortes de cette première enfance" (Freud, 1939/2001, p. 159).

$2^{\circ}$ Résistance défensive car, dans 1'idéal de la "talking cure", le travail d'élaboration et l'enforme représentationnel par les images et les mots doit aboutir à la perlaboration, fussent-ils longs et difficiles (Freud, 1914/1953, p. 115).

Mais ce fragment clinique, issu d'un travail d'analyse des pratiques, exige une ouverture de transfert, bien plus grande que celle que nous venons d'effectuer; et ce, même si la position, issue du transfert et de la rencontre toujours inédite avec un patient (Chaumon, 2009, 110) — ou une équipe (Cabassut, 2017), nous permet l'invention d'une "fiction de transfert" (Ibid.) permettant la relance du désir et de l'amour dans le travail personnel et institutionnel de chacun, comme de tous. En d'autres termes, la scène de la supervision offre au soignant le passage d'une posture de clivage sado-masochiste propre à la passion mélancolique du lien, à celle d'un amour de transfert qui implique la division hainamorative. Nous retombons là, sur la dimension du Sujet de l'Individuel et du Collectif initialement abordé, cette fois, non plus dans le lien autisme et psychanalyse, mais dans celui que nous offrent les apports de la psychothérapie institutionnelle. Tosquelles, Oury et quelques autres en effet, ne cessent de traiter du "contre-transfert institutionnel", soit ce qui de la souffrance des populations accueillies, ne cesse pas d'échouer, de se transporter auprès des équipes soignantes, et nécessite donc son perpétuel traitement. C'est dire si, dans l'accompagnement [éducatif, social, thérapeutique, etc.] et le quotidien auprès des dits autistes, dans la confrontation à cet archaïsme d'une souffrance mutique qui rentre peu ou pas dans les mots, la scène du travail de supervision [Analyse des pratiques, Régulation d'équipe, Analyse Institutionnelle] s'avère essentielle. Elle dessine le projet humain et politique, celui d'un vivre-ensemble qui ne soit pas de type ségrégatif, fondé donc sur une ligne de démarcation entre ceux diagnostiqués TSA et les autres; elle en détermine également ses fondements dans la pratique: le transfert est toujours entre deux; impossible de le partager [sauf imaginairement] avec le collègue, le médecin, les parents, etc. Par contre, il reste possible de collectiviser son analyse (Cabassut, 2017), ce qui n'est pas sans produire son effet thérapeutique, tant dans la relation clinique qu'institutionnelle. Rappelons que le premier sens du mot "thérapeute" est "celui qui prend soin" (Ouaknin, 1994, p. 13).

La scène de la supervision permet de restaurer l'illusion fondamentale perdue de l'amour et du désir, et avec elle celle de la transitionnalité. 


\section{ARTIGOS}

Le paradoxe winnicottien caractéristique de la souffrance autistique - soit ni avec, ni sans l'Autre —, est alors illusoirement soutenu: il n'a pas à être résolu et autorise le passage de l'identification imaginaire à l'identification symbolique et donc de l'impuissance à l'impossible. Son absence, au contraire, libère Thanatos, lequel fait retour de façon funèbre dans le lien social comme dans la clinique. ${ }^{22} \mathrm{Il}$ s'avère particulièrement prégnant dans les débats passionnels sur l'autisme où il est toujours question d'exclure l'Autre [parental, analytique, psychiatrique, etc.] et non de les mettre en dialectique. Les élans maniaques et "créatifs" du sujet [multiplication des plans Autisme, forums, documentaires à charge, etc.] viseraient, selon nous, à se défendre contre les effets objectivants de la pulsion de mort. Si dans l'autisme, le stade du miroir proposé par Lacan n'est pas structurant pour le sujet, il tend néanmoins à être désubjectivant pour l'Autre. ${ }^{23} \mathrm{La}$ souffrance autistique rend opaque la place spontanément occupée dans le lien d'altérité et complique l'interprétation de la demande de transfert. L'anesthésie du désir du soignant ne peut donc que laisser place à la technicisation et la planification dans la prise en charge de l'autisme: rééducation du Moi au détriment de l'accueil de la subjectivité. Cette anesthésie du désir de l'Autre, qui est aussi celui du sujet, est l'effet principal du discours capitaliste dans les (dé) constructions cette fois des subjectivités, dans le rapport immédiat à la jouissance. Répétons-le: forclusion de la castration, rejet des choses de l'amour et des affaires du sexe (Lacan, 1973/1981) Le discours capitaliste potentialise la mélancolie du soignant. La conception du soin de la souffrance autistique se modèle sur "l'état d'urgence", lequel n'est autre qu'un état d'exception; mais lorsque celui-ci tend à devenir la règle au commandement du lien social et du vivre ensemble, s'ouvre alors un espace propice au camp (Agamben, 1995): serions-nous dans un plan d'extermination du désir du soignant?

La restauration d'une clinique de l'amour et du désir, nous offre donc une alternative à la conception actuelle du DSM5 et du TSA, telle que d'aucuns, tenant d'une "psychologie scientifique" ou se référant au discours de la science dans son alliance à celui du marché (Lapeyre \& Sauret, 2000), produisent une

22 "La marque d'un objet non advenu au statut d'objet-tiers-manquant. N'est-ce pas ce qui rapproche la mélancolie de ces temps de passion où le funèbre semble se profiler derrière la jubilation et la jouissance?" (Hassoun, 1992, p. 108).

${ }^{23}$ Sur ce point se reporter à Marie-Christine Laznik (2014). Un psychanalyste avec les parents. Paris: Erès. 
caricature de l'approche psychanalytique, pour au mieux en faire un déterminisme pathologique obsolète, au pire, une imposture ridicule. ${ }^{24}$ Plutôt que de troubles neuro-cognitifs et de remédiations postmodernes qu'elles déterminent via les différentes "méthodes" de conditionnement comportemental, si décriées par bon nombre de récits de sortie d'autismes, ${ }^{25}$ nous préférons nous laisser enseigner par notre pratique auprès d'eux, et ce dans le sillage freudien. Il faut dire que la psychanalyse n'établit pas de différence de nature entre le normal et le pathologique, mais seulement d'intensité ou de degré. Il faut dire également, que si l'une se sert de la mise en lumière de la normalité par l'énigme de la maladie mentale et/ou de la folie, l'autre tend à produire une norme dont l'écart-type à celle-ci suffit à désigner le pathologique. Aussi, c'est bien la folie qui, tel un miroir grossissant de l'être, dicte la "norme" de la normalité et non plus l'inverse, celle d'un diktat éthico-moral variable selon les époques et les discours dominants, auxquelles les récentes batailles cliniques et socio-politiques de l'autisme nous assignent: en ce 8 décembre 2016, la proposition de loi du député Fasquelles [appuyée par 92 autres parlementaires!] proposait d'interdire la psychanalyse et le packing, qualifiés de pratiques maltraitantes dans la prise en charge de l'autisme, a été rejetée. ${ }^{26}$ Jusqu'à quand?

\section{Références}

Agamben,G. (1995). Qu'est-cequ'un camp? In Moyens sans fins/Notes sur la politique (pp. 47-56). Paris:Payot e Rivages, rivages poche/Petite bibliohèque.

André, S. (2015). Le sens de l'holocauste: jouissance et sacrifice. Bruxelles, Belgique: Editions le Bord de l'eau. Collection la Muette.

${ }^{24}$ Se reporter à la conférence de Joël Swedsen du 10 janvier 2018 "Regard scientifique sur la psychanalyse" (https://www.youtube.com/watch?v=RBd2sm-0eyM)

${ }^{25}$ Se reporter à Maleval (2009) cité en références.

${ }^{26}$ Le SIEEURPP (Séminaire Inter-Universitaire Européen d'Enseignement et de Recherche en Psychopathologie et Psychanalyse) mentionne qu'une bataille a été gagnée, mais que la guerre va hélas! continuer... L'interdiction et le risque de sanction pénale en cas d'utilisation de la méthode psychanalytique, ne sont-ils pas la prémisse de la logique ségrégative propre à cette médecine "scientifique" d'état, qui nous renvoie à des heures sombres de l'histoire? Nous faisons référence à la non moins "euthanasie d'état" qui aura rendue possible le gazage des malades mentaux par le régime nazi via le programme d'extermination $\mathrm{T} 4$. 


\section{ARTIGOS}

Anzieu, D. (1981). Le groupe et l'inconscient. L'imaginaire groupal. Paris, France: Dunod.

Benslama, F. (2014). La guerre des subjectivités en Islam. Paris, France: Ed. Lignes.

Cabassut, J. (2017). “Bonjour l'institution!” [Formation, Supervision et pratiques en clinique institutionnelle: Freud, Marx, Lacan, Oury, Tosquelles et quelques autres]. Nîmes, France: Champ Social Editions.

Chaumon, C. (2009). Henri Ray-Flaud, L'enfant qui s'est arrêté au seuil du langage. Comprendre l'autisme. Essaim, 1(22), 109-111.

Freud, S. (2001). L'homme-Moïse et la religion monothéiste. Paris, France: Gallimard, Folio-Essai. (Travail original publié dans 1939).

Freud, S. (1953). Remémoration, Répétition et Perlaboration. In La technique psychanalytique (pp. 105-115). Paris, France: PUF. (Travail original publié dans 1914).

Haag, G. (1995). Identification adhésive, identité adhésive, adhésivité. Vocabulaire de psychanalyse groupale et familiale (Tome1). Paris, France: Editions du College de Psychanalyse Groupale et Familiale.

Hassoun, J. (1992). La cruauté mélancolique. Paris, France: Champs-Flammarion.

Khon, M. (1999). Acte narratif et cas. In Le cas en controverse (pp. 51-58). Sous la direction de P. Fédida et F. Villa. Paris, France: PUF.

Klein, M. (1968). À propos de l'identification. Envie et gratitude et autres essais. Paris, France: Gallimard. (Travail original publié dans 1955).

Lacan, J. (1991). Le séminaire. Livre XVII. L'envers de la psychanalyse. Paris, France: Seuil. (Travail original publié dans 1969-1970).

Lacan, J. (1971-1972). Le savoir du psychanalyste. Entretiens de Sainte Anne. Inédit, Leçon du 6 janvier 1972.

Lapeyre, M., Sauret, M-J. (2000). Lacan: le retour à Freud. Paris, France: Editions Milan, Coll. Les Essentiels Milan.

Laznik, M-C. (2014). Un psychanalyste avec les parents. Paris, France: Erès.

Maleval, J. C. (2009). L'autiste et sa voix. Paris, France: Seuil-Champ Freudien.

Menard, A. (2008). Voyage au pays des psychoses. Nîmes, France: Champ social Ed.

Ouaknin, M.-A. (1994). Bibliothérapie. Lire, c'est guérir. Paris, France: Seuil.

Pépin, C. (2018). Pourquoi avons-nous besoin d'être aimé? Philosophie Magazine, 116, 16, Février 2018.

Ricœur, J-P. (2007). Lacan, l'amour. Psychanalyse, 3(10), 5-32. DOI 10.3917/ psy.010.0005.

Rouzel, J. (2018). La folie douce/Psychose et création. Préface de J. Cabassut. Toulouse, France: Erès. 
Rouzel, J. (2013). La prise en compte des psychoses dans le travail éducatif. Toulouse, France: Erès.

Sauret, M-J (2008). Mélancolie et lien social. Essaim, 1(20), 52-72. 'L'exception mélancolique".

Sauret, M-J. (2009). Malaise dans le capitalisme. Toulouse, France: Presses Universitaires du Mirail, Psychanalyses \&.

\section{Resumés}

(O drama do autismo: entre o amor e o desejo)

A partir de sua práxis, os autores propõem questionar o impensável da problemática autista a partir do amor e do desejo, que é seu grande veículo. Para tanto, propõem relacionar o sujeito do sujeito e o sujeito do coletivo, a formação da multidão e a paixão melancólica para apreender as lições sobre os efeitos estruturantes do chamado sujeito autista em seus relatos para o Outro, e vice-versa, sobre a qualidade do vínculo social contemporâneo. Eles argumentam que a conjuntura entre a questão autista e o discurso capitalista potencializa a melancolia do cuidador [espelhando a melancolia do Outro materno]. A atual concepção de cuidado, que exclui a especificidade do amor da transferência específica ao sofrimento autista, destrói qualquer ideia de envolvimento do profissional no encontro. Portanto, apenas a restauração das dimensões do amor à transferência e do desejo, como o tratamento de seus incuráveis, possibilita pensar elou repensar as práticas atuais, desta vez a partir da ética da clínica psicanalítica.

Palavras-chave: Amor, desejo, autismo, vínculo social

(The drama of autism: between love and desire)

Based on their praxis, the authors propose to question the unthinkable of the autistic problematic based on love and desire, which is its great vehicle. To this end, they propose to relate the subject of the individual and the subject of the collective, the formation of the crowd and melancholic passion, in order to grasp the lessons on the structuring effects of the so-called autistic subject in their reports. To the Other, and vice versa, about the quality of the contemporary social bond. They argue that the conjuncture between the autistic problem and the capitalist discourse potentiates the caregiver's melancholy [mirroring the melancholy of the maternal Other]. The current conception of care, which excludes the specificity of the love from the specific transference to the autistic suffering, destroys any idea of involvement of the professional in the encounter. Therefore, only the restoration of the dimensions of love to transference and of desire, as the treatment of their incurable, make it 


\section{ARTIGOS}

possible to think and/or to rethink current practices, this time based on the ethics of the psychoanalytical clinic.

Key words: Love, autism, desire, social bond

(El drama del autismo: entre el amor y el deseo)

Desde su praxis, los autores proponen cuestionar lo impensable de la problemática autista a partir del amor y del deseo, que es su principal vehículo. Para ello, proponen relacionar el sujeto del individuo y el sujeto del colectivo, la formación de la multitud y la pasión melancólica, y con esto, aprender las lecciones sobre los efectos estructuradores del llamado sujeto autista en sus relaciones, con el Otro y viceversa, sobre la calidad del vínculo social contemporáneo. Los autores argumentan que la coyuntura entre el problema autista y el discurso capitalista intensifica la melancolía del cuidador (reflejando la melancolía del Otro materno). La actual concepción de cuidado, que excluye la especificidad del amor de transferencia específico del sufrimiento autista, destruye cualquier idea de participación del profesional en el encuentro. Por lo tanto, solamente la restauración de las dimensiones del amor de transferencia y del deseo, así como el tratamiento de sus incurables, nos permiten repensar y/o reconsiderar las prácticas actuales, esta vez a partir de la ética de la clínica psicoanalítica.

Palabras clave: Amor, autismo, deseo, vínculo social

(Das Drama des Autismus: zwischen Liebe und Verlangen)

Basierend auf ihrer Praxis schlagen die Autoren vor, das Undenkbare des autistischen Problems in Frage zu stellen, ausgehend von der Liebe und vom Wunsch, welcher sein wichtigstes Vehikel ist. Zu diesem Zweck schlagen sie vor, eine Beziehung herzustellen zwischen dem Subjekt des Individuellen und dem Subjekt des Kollektiven, der Bildung der Masse und der melancholischen Leidenschaft, was dazu dient, die Lehren zu erfassen über die strukturierenden Wirkungen des sogenannten autistischen Subjekts in seinen Beziehungen mit dem Anderen und vice versa über die Qualität der gegenwärtigen sozialen Bindung. Die Autoren argumentieren, dass die Konjunktur zwischen dem autistischen Problem und dem kapitalistischen Diskurs die Melancholie der Betreuer potenziert (was wiederum die Melancholie des mütterlichen Anderen widerspiegelt). Die gegenwärtige Auffassung von Pflege, die die Besonderheit der Liebe der spezifischen Übertragung auf autistisches Leiden ausschließt, zerstört jede Vorstellung von einer Beteiligung des Berufstätigen an der Begegnung. Allein die Wiederherstellung der Dimensionen der Übertragungsliebe und des Wunsches, als Behandlung ihrer Unheilbaren, ermöglicht ein Umdenken und/oder ein Überdenken der derzeitigen Praktiken, nun basierend auf der Ethik der psychoanalytischen Klinik.

Schlüsselwörter: Liebe, Autismus, Wunsch, Soziale Bindung

Rev. Latinoam. Psicopat. Fund., São Paulo, 21(4), 697-714, dez. 2018 
Citação/Citation: Cabassut, J., Barriol, C. (2018, dezembro). Le drame de l'autisme: entre amour et désir. Revista Latinoamericana de Psicopatologia Fundamental, 21(4), 697-714. http://dx.doi.org/10.1590/1415-4714.2018v21n4p697.2.

Editora da entrevista/Editor: Profa. Dra. Ana Maria G. R. Oda e Profa. Dra. Sonia Leite.

Recebido/Received: 14.6.2018 / 6.14.2018 Aceito/Accepted: 18.8.2018 / 8.18.2018

Copyright: (C) 2009 Associação Universitária de Pesquisa em Psicopatologia Fundamental/ University Association for Research in Fundamental Psychopathology. Este é um artigo de livre acesso, que permite uso irrestrito, distribuição e reprodução em qualquer meio, desde que o autor e a fonte sejam citados / This is an open-access article, which permits unrestricted use, distribution, and reproduction in any medium, provided the original authors and sources are credited.

Financiamento/Funding: Este trabalho não recebeu financiamento / This work received no funding.

Conflito de interesses/Conflict of interest: Os autores declaram que não há conflito de interesses / The authors declare that there is no conflict of interest.

\section{Jacques Cabassut}

Professeur de psychopathologie clinique et psychanalyse, Université Nice Sophia Antipolis (Nice, France); Membre de l'Université Côte d'Azur (UCA), EA 3159 - Laboratoire Interdisciplinaire Récits, Cultures Et Sociétés (Nice, France).

Département de Psychologie

Faculté des Lettres, Arts et Sciences Humaines - Campus Carlone

98 bd Edouard Herriot

BP 3209

06204 NICE Cedex 3, France

cabassut@unice.fr

\section{Céline Barriol}

Maître de Conférences en Psychologie clinique du développement, Université Nice Sophia Antipolis (Nice, France); Membre de l'Université Côte d'Azur (UCA), EA 3159 Laboratoire Interdisciplinaire Récits, Cultures Et Sociétés (Nice, France).

Département de Psychologie

Faculté des Lettres, Arts et Sciences Humaines - Campus Carlone

98 bd Edouard Herriot

BP 3209

06204 NICE Cedex 3, France

barriol@unice.fr

This is an open-access article, which permits unrestricted use, distribution, and reproduction in any medium for non-commercial purposes provided the original authors and sources are credited. 